\title{
Dipivefrin Hydrochloride
}

National Cancer Institute

\section{Source}

National Cancer Institute. Dipivefrin Hydrochloride. NCI Thesaurus. Code C47495.

An ester with sympathomimetic activities. Dipivefrin hydrochloride is a prodrug of epinephrine that, due to its greater lipophilicity, allows for better penetration into the anterior chamber. Once inside the eye, dipivefrin hydrochloride is converted by hydrolysis to epinephrine. Epinephrine enhances the outflow of aqueous humor and decreases the production of aqueous humor by vasoconstriction. This leads to a reduction in intraocular pressure. 